\title{
Aportes a LA COLECCIÓN DE HONGOS LIQUENIZADOS DEL HERBARIO dEL INSTITUTO de BotánICA Carlos Spegazzini (LPS)
}

\author{
JUAN M. LAVORNIA ${ }^{1,2}$, RENATO A. GARCÍA ${ }^{1,3}$, VILMA G. ROSATO ${ }^{1,3,4}$, MARÍA J. \\ KRISTENSEN ${ }^{2,5}$, JORGE A. CHAYLE $^{7}$ y MARIO N. SAPARRAT ${ }^{1,6,7}$
}

\begin{abstract}
Summary: Contributions to the collection of liquenized fungi from the herbarium of the Institute of Botany Carlos Spegazzini (LPS). The Institute of Botany Carlos Spegazzini (IBCS) (UNLP, La Plata) hosts an herbarium of fungi (LPS) of approximately 40,000 specimens, with 4200 type specimens. The aim of this study was to examine the specimens of lichenized fungi deposited in the IBCS, update their taxonomy and name, check questionable species determination, identify those not certain, and incorporate them into the Herbarium LPS. 192 specimens were studied coming from 11 Provinces of Argentina, as well as Brazil, Uruguay and France and they were determined based on their exo-morphology, histological and histochemical reactions. Thin Layer Chromatographs (TLC) were also performed to study the secondary metabolites present. Argentinean geographical distribution of the identified species was established. The $91.66 \%$ of the materials examined (176 specimens) was corrected and accounted for a total of 91 species, 50 genera and 21 families, with Parmeliaceae (16 genera, 31 species), Graphidaceae $(4 ; 5)$ and Physciaceae $(3 ; 9)$ as the best represented. The name of 56 specimens belonging to 32 species was updated. The identity of 120 specimens was modified to species level (87), genera (33) and family (1). The distribution of 9 species in Argentina was expanded.
\end{abstract}

Key words: Fungi, Herbarium LPS, Lichens, Argentina.

Resumen: El Instituto de Botánica Carlos Spegazzini (IBCS) (UNLP, La Plata) contiene un herbario de hongos (LPS) de aproximadamente 40000 ejemplares, con 4200 especies tipo. El objetivo de este trabajo fue examinar los ejemplares de hongos liquenizados depositados en el IBCS, actualizar su taxonomía y denominación, revisar las especies con determinación dudosa, identificar aquellos no determinados, e incorporarlos en el Herbario LPS. Se estudiaron 192 ejemplares de 11 provincias de Argentina y de Brasil, Uruguay y Francia y de se determinaron en base a su exo-morfología, cortes histológicos y reacciones histoquímicas. También se realizaron Cromatografías en Capa Delgada (TLC) para estudiar los metabolitos presentes. Se estableció la distribución geográfica en Argentina de las especies identificadas. Se corrigió el $91,66 \%$ de los materiales examinados (176 ejemplares), contabilizándose un total de 91 especies, 50 géneros y 21 familias, siendo las mejor representadas Parmeliaceae (16 géneros; 31 especies), Graphidaceae $(4 ; 5)$ y Physciaceae (3; 9). Se actualizó la nominación de 56 ejemplares pertenecientes a 32 especies. Se modificó la identidad de 120 ejemplares, a nivel de especie (87), género (33) y familia (1). Se amplía la distribución en Argentina de 9 especies.

Palabras clave: Fungi, herbario LPS, Líquenes, Argentina.

\footnotetext{
${ }^{1}$ CONICET

${ }^{2} \mathrm{CINEA}, \mathrm{FCH}$, UNICEN, Tandil, Buenos Aires, Argentina.

${ }^{3}$ LEMIT, CIC-PBA La Plata, Buenos Aires, Argentina.

${ }^{4}$ UTN, FRLP, La Plata, Buenos Aires, Argentina.

5IGS-CISAUA, UNLP, La Plata, Buenos Aires, Argentina.

${ }^{6}$ Instituto de Fisiología Vegetal, UNLP, La Plata, Buenos Aires, Argentina.

${ }^{7}$ Instituto de Botánica Spegazzini, FCNYM, UNLP, La Plata, Buenos Aires, Argentina.

* Corresponding autor. E-mail: juan_lavornia@hotmail.com
} 


\section{INTRODUCCIÓN}

El Instituto de Botánica C. Spegazzini (IBCS), que pertenece a la División Científica del Museo de Ciencias Naturales, Facultad de Ciencias Naturales y Museo de la Universidad Nacional de La Plata, es sede de un herbario conocido e identificado nacional e internacionalmente con el acrónimo LPS a partir de su inclusión en el Index Herboriarum en 1937.

En el Herbario LPS se conservan cuerpos fructíferos de hongos, líquenes y sustratos fuentes de hongos, así como también otros grupos entre los que se destaca la colección de briófitos. Entre éstos, se encuentran materiales tipo, tales como los estudiados por el prestigioso micólogo Carlos Spegazzini, que están acompañados con descripciones, fotografías, preparados para microscopía y/o ilustraciones a mano alzada realizadas y legadas, por lo que la colección constituye una herramienta de referencia indispensable para los trabajos científicos y presenta relevancia histórica a la vez que forma parte del patrimonio cultural local. Además el LPS integra el proyecto de la Red Nacional de Colecciones Biológicas (RNCB, Argentina) para la organización Internacional Global Biodiversity Information Facility (GBIF) [http://www.gbifargentina.org.ar/] desde 2004.

La colección del herbario se inició en el año 1935 con los especímenes legados por C. Spegazzini y posteriormente se vio ampliada por la contribución de otros investigadores como E. Horak, F. Petrak, R. Singer, W. G. Solheim y J. E. Wright. El herbario cuenta con 40000 ejemplares y 4200 materiales tipo, ordenados por género siguiendo a Clements \& Shear (1931). De ellos, 12000 provienen de canje y 300 son inéditos. Aunque los materiales conservados en el LPS son mayoritariamente hongos no liquenizados, existe en el IBCS un gran volumen de material liquénico proveniente de colectas realizadas en todo el país y países limítrofes, de intercambio con otras instituciones y de donaciones de naturistas, que aún debe identificarse, cuantificarse e incorporarse al Herbario LPS. Además, los cambios vigentes en la sistemática de los Ascomycota y Basidiomycota liquenizados, determinan la necesidad de revisar la nomenclación del material liquénico por incorporar al LPS y -en los casos que haga falta- corregirla de acuerdo a su identidad o posición sistemática actual. Estas tareas son prioritarias en la gestión y conservación de las colecciones biológicas y para la puesta en valor de este patrimonio biológico y cultural.

El objetivo de este trabajo fue examinar los ejemplares de hongos liquenizados depositados en el IBCS, actualizar su taxonomía y denominación, revisar las especies con determinación dudosa, identificar aquellos no determinados, e incorporarlos en el Herbario LPS. En esta contribución se dan a conocer los avances obtenidos en el marco de la pasantía "Determinación de ejemplares (hongos liquenizantes) de la colección del Instituto de Botánica Carlos Spegazzini" (Resolución Comisión de Posgrado, FCNyM, UNLP del 09/09/2013, Fs. 527-531) realizada por el Dr. J. M. Lavornia y el Lic. R. A. García en el IBCS durante 2014, en la que trabajaron con material que se encontraba en el IBCS a la espera de ser incorporado al LPS. Se presenta un listado de las especies que representan novedades, incluyendo su distribución geográfica en Argentina.

\section{Materiales y Métodos}

Se relevó una tercera parte $(n=192)$ de los ejemplares de la colección del IBCS que no estaban ingresados al Herbario LPS. Entre éstos se incluían materiales no determinados y unos con una identificación dudosa. Se procedió a examinar cada ejemplar, determinando o verificando su identidad taxonómica $\mathrm{y}$, cuando necesario, actualizando su denominación, para luego incorporarlos en el Herbario LPS. Se estudiaron ejemplares pertenecientes a 11 provincias de Argentina (predominantemente Tierra del Fuego, Buenos Aires y Chubut) y material de Brasil, Uruguay y Francia. Para la identificación de las especies se analizó su exo-morfología bajo lupa estereoscópica $\mathrm{y}$ se hicieron reacciones y pruebas en sectores específicos de los talos con $\mathrm{KOH} 0,5 \%, \mathrm{NaClO}$ y luz ultravioleta (Brodo, 2003). También se realizaron cortes histológicos de talo a mano alzada para observar las estructuras internas bajo microscopio óptico. Se utilizaron las claves de identificación de Adler (1992), Scutari (1992), Kashiwadani \& Kalb (1993), Galloway (1992, 1994), Marcano \& Morales (1994), Sipman (2005) y Messuti \& De La Rosa (2009). En los casos en que 
fue necesario, se utilizó la técnica de cromatografía en capa delgada (Culberson, 1972; Culberson \& Amman, 1979) utilizando instrumental del Laboratorio de Entrenamiento Multidisciplinario para la Investigación Tecnológica (LEMIT).

Se revisó la distribución de las especies de acuerdo a Calvelo \& Liberatore (2002), Adler \& Calvelo (2010), Estrabou et al. (2006), Messuti \& De La Rosa (2009), Michling \& Ferraro (2012a, b), Adler (2013), Michling (2014) y Rodríguez et al. (2016). Para cada especie se consignó la distribución en las provincias de: Buenos Aires (BA), Catamarca (CA), Chaco (CH), Chubut (CHU), Córdoba (COR), Corrientes (CO), Entre Ríos (ER), Islas Malvinas y del Atlántico Sur (IM y AS), Jujuy (JU), La Pampa (LP), La Rioja (RI), Mendoza (ME), Misiones (MI), Neuquén (NE), Río Negro (RN), Salta (SA), Santa Cruz (SC), Santiago del Estero (SE), Santa Fé (SF), Tucumán (TU) y Tierra del Fuego (TF). La denominación del material se actualizó según los catálogos web Index Fungorum [http://www.indexfungorum.org] y Mycobank [www.mycobank.org].

\section{Resultados}

El relevamiento realizado durante la pasantía permitió identificar 91 especies pertenecientes a 50 géneros y 21 familias. Parmeliaceae fue la familia mejor representada con 16 géneros y 31 especies, seguida de Graphidaceae con 4 géneros y 5 especies, y Physciaceae con 3 géneros y 9 especies (Tablas 1 y 2).

De los 192 ejemplares revisados, la nominación de 176 fue modificada, siendo actualizada en el caso de 56 materiales (Tabla 2) y registrada en 120 materiales (87 a nivel de especie, 33 a nivel de género y un ejemplar que sólo pudo ser determinado a nivel de familia; Tabla 1). A pesar de corresponder a materiales con datos de registro, sólo el 59,65\% presentó lugar de colección.

Se proporciona a continuación el listado de once especies para las cuales se amplía la distribución en Argentina.

Crespoa crozalsiana (B. de Lesd. ex Harm.) Lendemer \& B.P. Hodk., N. Amer. Fung. 7(2): 3. 2013. Citada previamente en BA, CA, CO, COR, ER, JU, MI, SE, TU.

Material estudiado: ARGENTINA. Prov.
Chubut: Río Arrayanes, 1996, Martínez 48726 (LPS).

Flavoparmelia soredians (Nyl.) Hale, Mycotaxon 25(2): 605. 1986. Citada previamente para BA, CA, CHU, CO, SL.

Material estudiado: ARGENTINA. Prov. La Pampa: Lihuel-Calel, 1952, Ragonese y Ciccesini 48719 (LPS).

Haematomma chilena C.W. Dodge, Nova Hedwigia 12: 343. 1967. Citada previamente para CHU, NE, RN.

Material estudiado: ARGENTINA. Prov. Tierra del Fuego: Valle de Olivia, 1986, N. Alboff 48725 (LPS).

Physcia aipolia (Humb.) Fürnr., Naturhist. Topogr. Regensburg: 249. 1839. Citada previamente para BA, CA, CO, LP, MI, SL, TU.

Material estudiado: ARGENTINA. Prov. Entre Ríos: Concordia, 1967, E. Speg. 48718 (LPS).

Psora icterica (Mont.) Müll. Arg., Flora (Regensburg) 71(3): 45. 1888. Citada previamente para BA, CO, COR, ME, RN.

Material estudiado: ARGENTINA. Prov. Jujuy: Mina Aguilar, 1948, A. L. Cabrera 48727 (LPS).

Punctelia microsticta (Müll.Arg.) Krog, Nord. J. Bot. 2: 291. 1982. Citada previamente para BA, $\mathrm{CH}, \mathrm{CO}, \mathrm{JU}, \mathrm{MI}, \mathrm{RI}, \mathrm{SA}, \mathrm{SF}, \mathrm{SE}, \mathrm{TF}$.

Material estudiado: ARGENTINA. Prov. Chubut: Lago Puelo, 1966, Martínez 48720 (LPS). Prov. Tucumán: Tafí del Valle, 1949, A. Martínez s/ $\mathrm{n}^{\circ}$ (LPS).

Umbilicaria polyrrhiza (L.) Fr., Sched. Crit. Lich. Suec. Exsicc. 5-6: 3. 1825. Citada previamente para IM y AS, TF.

Material estudiado: ARGENTINA. Prov. Chubut: Lago Blanco, 1903, C. Bettfreund 48728 (LPS).

Ramalina celastri (Spreng.) Krog \& Swinscow, Nord. J. Bot. 23: 159. 1976. Citada previamente para BA, CA, CO, COR, ER, JU, MI, SA.

Material estudiado: ARGENTINA. Prov. Chaco: Bermejo, 1900, Kernes 48910 (LPS). ARGENTINA. Prov. Tierra del Fuego: Islas de los Estados, 1902, L. Rodríguez 48920 (LPS). ARGENTINA. Prov. Tucumán: Tucumán, 1900, Kernes 48730 (LPS).

Pseudocyphellaria aurata (Ach.) Vain., Acta Soc. Fauna Flora Fenn. 7(1): 183. 1890. Citada previamente para RN, TU, TF.

Material estudiado: ARGENTINA. Prov. 
Bol. Soc. Argent. Bot. 52 (1) 2017

Tabla 1. Ejemplares del IBCS revisados $(n=120)$, determinados e ingresados al Herbario LPS.

\begin{tabular}{|c|c|c|c|}
\hline Familia & Especie & Identidad anterior & $\begin{array}{l}\text { Ejemplares } \\
\left(n^{\circ}\right)\end{array}$ \\
\hline \multirow[t]{36}{*}{ Parmeliaceae Zenker } & Crespoa carneopruinata (Zahlbr.) Lendemer \& B.P. Hodk. & Parmelia & 1 \\
\hline & $\begin{array}{l}\text { Crespoa crozalsiana (B. de Lesd. ex Harm.) Lendemer } \\
\text { \& B.P. Hodk. }\end{array}$ & Parmelia & 1 \\
\hline & Flavoparmelia soredians (Nyl.) Hale & Parmelia & 1 \\
\hline & Hypogymnia lugubris (Pers.) Krog & Parmelia & 1 \\
\hline & Hypotrachyna (Vain.) Hale sp. & Parmelia & 1 \\
\hline & Hypotrachyna brevirhiza (Kurok.) Hale & Parmelia & 3 \\
\hline & Hypotrachyna osorioi (Hale) Hale & Parmelia cetrata & 1 \\
\hline & $\begin{array}{l}\text { Hypotrachyna subgen. Everniastrum (Hale ex Sipman) } \\
\text { Divakar, A. Crespo, Sipman, Elix \& Lumbsch }\end{array}$ & Parmelia & 1 \\
\hline & $\begin{array}{l}\text { Hypotrachyna vexans (Zahlbr. ex W.L. Culb. \& C.F. Culb.) } \\
\text { Divakar, A. Crespo, Sipman, Elix \& Lumbsch }\end{array}$ & Evernia furfuracea & 1 \\
\hline & Menegazzia A. Massal. sp. & $\begin{array}{l}\text { Evernia aff. } \\
\text { fusfuracea }\end{array}$ & 1 \\
\hline & Menegazzia A. Massal. sp. & Parmelia & 7 \\
\hline & Neuropogon trachycarpus Stirt. & Usnea & 1 \\
\hline & Parmotrema A. Massal. sp. & Parmelia & 3 \\
\hline & Parmotrema pilosum (Stizenb.) Krog \& Swinscow & Parmelia & 1 \\
\hline & Parmotrema pilosum (Stizenb.) Krog \& Swinscow & Imbricaria laevigata & 1 \\
\hline & Parmotrema reticulatum (Taylor) M. Choisy & Parmelia cetrata & 1 \\
\hline & Parmotrema tinctorum (Despr. ex Nyl.) Hale & Imbricaria tiliacea & 1 \\
\hline & Parmotrema tinctorum (Despr. ex Nyl.) Hale & Parmelia & 1 \\
\hline & Parmotrema uruguense (Kremp.) Hale & Parmelia & 1 \\
\hline & $\begin{array}{l}\text { Parmotrema ventanicum (Adler \& Elix) O. Blanco, A. } \\
\text { Crespo, Divakar, Elix \& Lumbsch }\end{array}$ & Parmelia & 1 \\
\hline & Punctelia constantimontium Sérus. & Parmelia & 1 \\
\hline & Punctelia microsticta (Müll. Arg.) & Parmelia & 1 \\
\hline & Punctelia perreticulata (Räsänen) G. Wilh. \& Ladd & Parmelia lorentzii & 2 \\
\hline & Usnea alata Motyka & Usnea sulcata & 1 \\
\hline & Usnea alata Motyka & Sin determinar & 1 \\
\hline & Usnea angulata Ach. & Usnea sulcata & 2 \\
\hline & Usnea pusilla (Räsänen) Räsänen & Usnea simplex & 1 \\
\hline & Usnea strigosa (Ach.) Pers. & Usnea barbata & 1 \\
\hline & Usnea strigosa (Ach.) Pers. & Usnea meriodinalis & 1 \\
\hline & Xanthoparmelia rupicola (Lynge) Elix \& J. Johnst. & Parmelia & 2 \\
\hline & Xanthoparmelia (Vain.) Hale sp. & Parmelia & 1 \\
\hline & Xanthoparmelia cotopaxiensis T.H. Nash, Elix \& J. Johnst. & Parmelia conspersa & 1 \\
\hline & Xanthoparmelia hypopsila (Müll. Arg.) & Parmelia & 2 \\
\hline & Xanthoparmelia hypopsila (Müll. Arg.) & Parmelia conspersa & 2 \\
\hline & Xanthoria elegans (Link) Th. Fr. & Lecidea & 1 \\
\hline & Xanthoria parietina (L.) Beltr. & Parmelia & 1 \\
\hline
\end{tabular}


J. M. Lavornia et al. - Aportes a la colección de líquenes LPS

\begin{tabular}{|c|c|c|c|}
\hline Familia & Especie & Identidad anterior & $\begin{array}{c}\text { Ejemplares } \\
\left(n^{\circ}\right)\end{array}$ \\
\hline Roccellaceae Chevall. & Mazosia rotula (Mont.) A. Massal. & Opegrapha protula & 1 \\
\hline $\begin{array}{l}\text { Haematommataceae } \\
\text { Hafellner }\end{array}$ & Haemmatoma chilenum C.W. Dodge & Haemmatoma & 1 \\
\hline \multirow[t]{5}{*}{ Physciaceae Zahlbr. } & Heterodermia sp. & Parmelia & 1 \\
\hline & Physciaceae Zahlbr. sp. & Parmelia & 1 \\
\hline & Physcia aipolia (Ehrh. ex Humb.) Fürnr. & Parmelia & 1 \\
\hline & Physcia alba (Fée) Müll. Arg. & Parmelia & 1 \\
\hline & Physcia (Schreb.) Michx. sp. & Parmelia & 1 \\
\hline \multirow[t]{12}{*}{ Lobariaceae Chevall. } & Pseudocyphellaria Vain. sp. & Parmelia & 2 \\
\hline & Pseudocyphellaria glabra (Hook. f. \& Taylor) C.W. Dodge & Pseudocyphellaria & 2 \\
\hline & Pseudocyphellaria aurata (Ach.) Vain. & Pseudocyphellaria & 1 \\
\hline & $\begin{array}{l}\text { Pseudocyphellaria berberina (G. Forst.) D.J. Galloway \& } \\
\text { P. James }\end{array}$ & Pseudocyphellaria & 5 \\
\hline & Pseudocyphellaria clathrata (De Not.) Malme & $\begin{array}{l}\text { Pseudocyphellaria } \\
\text { aurata }\end{array}$ & 2 \\
\hline & Pseudocyphellaria coronata (Müll. Arg.) Malme & Sin determinar & 3 \\
\hline & Pseudocyphellaria faveolata (Delise) Malme & Pseudocyphellaria & 2 \\
\hline & Pseudocyphellaria glabra (Hook. f. \& Taylor) C.W. Dodge & Sin determinar & 7 \\
\hline & Pseudocyphellaria hirsuta (Mont.) Malme & Pseudocyphellaria & 2 \\
\hline & Pseudocyphellaria obvoluta (Sw.) Malme & Pseudocyphellaria & 2 \\
\hline & Sticta ainoae D.J. Galloway \& J. Pickering & Parmelia & 4 \\
\hline & Sticta ainoae D.J. Galloway \& J. Pickering & Pseudocyphellaria & 2 \\
\hline \multirow{3}{*}{$\begin{array}{l}\text { Umbilicariaceae } \\
\text { Chevall. }\end{array}$} & Umbilicaria Hoffm. sp. & Gyrophora diblonii & 1 \\
\hline & Umbilicaria polyrrhiza (L.) Fr. & $\begin{array}{l}\text { Parmelia } \\
\text { (Hypotrachyna) }\end{array}$ & 1 \\
\hline & Umbilicaria polyrrhiza (L.) Fr. & Parmelia & 1 \\
\hline \multirow{3}{*}{$\begin{array}{l}\text { Ramalinaceae } \\
\text { C. Agardh }\end{array}$} & Ramalina aspera Räsänen & Sin determinar & 1 \\
\hline & Ramalina celastri (Spreng.) Krog \& Swinscow & Ramalina ayumani & 1 \\
\hline & Ramalina celastri (Spreng.) Krog \& Swinscow & Sin determinar & 9 \\
\hline \multirow[t]{2}{*}{$\begin{array}{l}\text { Rhizocarpaceae M. } \\
\text { Choisy ex Hafellner }\end{array}$} & & & \\
\hline & Rhizocarpon geographicum (L.) DC. & Sin determinar & 1 \\
\hline Lecanoraceae Körb. & Lecanora Trevis. sp. & Parmelia & 1 \\
\hline $\begin{array}{l}\text { Nephromataceae } \\
\text { Wetmore ex J.C. } \\
\text { David \& D. Hawksw. }\end{array}$ & Nephroma Ach. sp. & Parmelia & 12 \\
\hline
\end{tabular}


Tabla 2. Listado de especies ingresadas al herbario LPS cuya nominación fue actualizada (n=56).

\begin{tabular}{|c|c|c|}
\hline Especies actualizadas en su nominación & Identidad anterior & $\begin{array}{c}\mathrm{N}^{\circ} \text { de } \\
\text { Ejemplares }\end{array}$ \\
\hline Bacidia millegrana (Taylor) Zahlbr. & Patellaria millegrana & 2 \\
\hline Byssoloma leucoblepharum (Nyl.) Vain. & Patellaria leucoblephora & 1 \\
\hline Calopadia perpallida (Nyl.) Vězda & Heterothecium perpallidum & 1 \\
\hline Caloplaca regalis (Vain.) Zahlbr. & Placodium regale & 1 \\
\hline Caloplaca saxicola (Hoffm.) Nordin & Placodium murorum & 2 \\
\hline Catillaria endoxantha (Müll. Arg.) Zahlbr. & Patellaria andoxantha & 1 \\
\hline Cryptothelium confluens (Müll. Arg.) Zahlbr. & Heufleria confluens & 1 \\
\hline Glyphis cicatricosa Ach. var. confluens (Zenker) Zahlbr. & Gliphys confluens & 1 \\
\hline Graphis virescens Müll. Arg. & Graphis virescens & 1 \\
\hline Hypogymnia lugubris (Pers.) Krog & Parmelia lugubris & 1 \\
\hline $\begin{array}{l}\text { Hypotrachyna cirrhata (Fr.) Divakar, Crespo, Sipman, Elix \& } \\
\text { Lumbsch }\end{array}$ & Parmelia cirrhata & 1 \\
\hline Hypotrachyna laevigata (Sm.) Hale & Parmelia laevigata & 1 \\
\hline Hypotrachyna sinuosa (Sm.) Hale & Parmelia sinuosa & 1 \\
\hline Lopezaria versicolor (Flot.) Kalb \& Hafellner & Patellaria versicolor & 1 \\
\hline Nephroma australe A. Rich. & Nephroma antarticum & 10 \\
\hline Nephroma lobuligerum (Müll. Arg.) Gyeln. & Nephromium lobuligerum & 1 \\
\hline $\begin{array}{l}\text { Parmotrema fistulatum (Taylor) O.Blanco, A.Crespo, Divakar, Elix } \\
\text { \& Lumbsch }\end{array}$ & Everniastrum fistulatum & 4 \\
\hline Patellaria chloritis (Tuck.) Müll. Arg. var. nigrata Müll. Arg. & Patellaria chorisis & 1 \\
\hline Phyllopsora parvifolia (Pers.) Müll. Arg. & Lecidea parvifolia & 1 \\
\hline Pleurosticta acetabulum (Neck.) Elix \& Lumbsch & Parmelia acetabulum & 1 \\
\hline Protousnea magellanica (Mont.) Krog & Usnea magellanica & 1 \\
\hline Pseudocyphellaria coronata (Müll. Arg.) Malme & Pseudocyphellaria endochrysa & 1 \\
\hline Pseudocyphellaria glabra (Hook. f. \& Taylor) C.W. Dodge & Pseudocyphellaria freycinetii & 1 \\
\hline Psora icterica (Mont.) Müll. Arg. & Lecidea icterica & 1 \\
\hline Punctelia hypoleucites (Nyl.) Krog & Imbricaria hypoleucites & 1 \\
\hline Punctelia microsticta (Müll. Arg.) Krog & Parmelia microsticta & 1 \\
\hline Ramalina celastri (Spreng.) Krog \& Swinscow & Ramalina ecklonii & 12 \\
\hline Sporopodium phyllocharis (Mont.) A. Massal. & Lopadium phyllocharis & 2 \\
\hline Trypethelium variolosum Ach. & Pseudopyrenula catervaria & 1 \\
\hline Xanthoparmelia conspersa (Ehrh. ex Ach.) Hale & Imbricaria conspersa & 1 \\
\hline Total ejemplares actualizados en su nominación & & 56 \\
\hline
\end{tabular}




\section{J. M. Lavornia et al. - Aportes a la colección de líquenes LPS}

Chubut: Río Arrayanes, 1966, A. Martínez 48926 (LPS). ARGENTINA. Prov. Jujuy: El Chucho, 1962, M. Ferrario 48925 (LPS). ARGENTINA. Prov. Jujuy: Tiraxis, 1962, M. Ferrario 48923 (LPS).

Sticta ainoae D.J. Galloway \& Pickering, Bibl. Lichenol. 38: 92. 1990. Citada previamente para CHU, NE, RN.

Material estudiado: ARGENTINA. Prov. Tierra del Fuego: Isla de los Estados, 1902, L. Rodríguez 48723 (LPS).

Xanthoparmelia cotopaxiensis T.H. Nash, Elix \& J. Johnst., Mycotaxon 28(2): 288. 1987. Citada previamente para CA, CHU, CO.

Material estudiado: ARGENTINA. Prov. Jujuy: Casabindo, 1948, A. L. Cabrera 48724 (LPS).

\section{Discusión Y ConClusiones}

Esta contribución representa un estado de avance en el registro de materiales de herbario depositados en el Herbario LPS que constituye información novedosa para la liquenobiota argentina. Puesto que se reporta el cambio en la denominación de 120 ejemplares, es notoria la importancia de esta actividad de acondicionamiento de materiales de herbario en el IBCS, su identificación e incorporación al acervo del Herbario LPS.

En relación a la distribución geográfica, los ejemplares analizados permitieron constatar que varias especies no estaban citadas para algunas provincias de Argentina, por lo que se amplió la distribución nacional de 11 especies. Para 2 especies de distribución amplia $-R$. celastri y $P$. aurata- se incorporaron citas en 2-3 provincias y se encontraron localidades intermedias para otras dos especies $-F$. soredians y $P$. aipolia-. Se constataron registros más australes de 3 especies -C. crozalsiana, H. chilena y $S$. ainoaey más septentrionales de otras tres $-P$. icterica y $U$. polyrrhiza y $X$. cotopaxiensis. Se resalta la existencia de mucho material inédito de las provincias de Tierra del Fuego y Chubut.

Los resultados obtenidos a partir del material analizado permiten suponer que la colección que alberga el IBCS a la espera de ingresar al LPS podría brindar mucha más información biogeográfica inédita, destacando el valor que presentan las colectas realizadas en el pasado por los colaboradores del Instituto como así también la necesidad de continuar este trabajo.

\section{Agradecimientos}

Se agradece la colaboración del personal del Instituto de Botánica Carlos Spegazzini y al revisor anónimo que mediante sus comentarios contribuyó a enriquecer y dar claridad al manuscrito.

\section{Bibliografía}

ADLER, M. T. 1992. Clave de los géneros y las especies de Parmeliaceae (Lichenes, Ascomycotina) de la provincia de Buenos Aires (Argentina). Bol. Soc. Argent. Bot. 28: 11-17.

ADLER, M. T. 2013. Líquenes Parmelioides (Parmeliaceae, Ascomycota) del Parque Nacional Copo (Provincia de Santiago del Estero, Argentina). Bol. Soc. Argent. Bot. 48: 387-406.

ADLER, M. T. \& S. CALVELO. 2007. Ampliación de las distribuciones de especies de Parmeliaceae (Ascomycota liquenizados) en la República Argentina. Bol. Soc. Argent. Bot. 42: 1-11.

ADLER, M. T. \& S. CALVELO. 2010. Flavoparmelia baltimorensis, Parmotrema yodae y Xanthoparmelia braziliensis, primeras citas para Argentina y ampliación de distribuciones para otras Parmeliaceae (Ascomycota liquenizados). Bol. Soc. Argent. Bot. 45: 5-16.

BRODO, I. M. 2003. Microchemical Methods for the Identification of Lichens. Bryologist 106: 345-345.

CULBERSON, C. F. 1972. Improved conditions and new data for identification of lichen products by standardized thin-layer chromatographic method. $J$. Chromatogr. 72: 113-125.

CULBERSON, C. F. \& K. AMMAN. 1979. Standar methode zur Dünnschictchromatographie von Flechtensubstanzen. Herzogia 5: 1-24.

CALVELO, S. \& S. LIBERATORE. 2002. Catálogo de los líquenes de la Argentina. Kurtziana 29: 7-170.

CLEMENTS, F. E. \& C. L. SHEAR. 1931. The genera of Fungi. H.W. Wilson Co., New York. Pp. 496.

ESTRABOU, C., RODRÍGUEZ, J. M., PRIERI, B. \& R. LIJTEROFF. 2006. Contribución al conocimiento de los macrolíquenes del extremo Sur del Gran Chaco (Argentina). Kurtziana 32: 25-43.

GALLOWAY, D. J. 1992. Studies in Pseudocyphellaria (lichens) III. The South American species. Bibl. Lichenol. 46: 1-275.

GALLOWAY, D. J. 1994. Studies on the lichen genus Sticta (Scheber) Ach.: I. Southern South American species. Lichenologist 26: 223-282. 
KASHIWADANI, H. \& K. KALB. 1993. The genus Ramalina in Brazil. Lichenologist 25: 1-31.

MARCANO, V. \& A. MORALES. 1994. Revisión del género Umbilicaria (Ascomicetes liquenizados) en Venezuela. Erstia 4: 21-35.

MESSUTI, M. I. \& I. N. DE LA ROSA. 2009. Notes on the genus Haematomma (Ascomycota, Lecanoraceae) in Argentina. Darwiniana 47: 297-308.

MICHLIG, A. 2014. Canoparmelia y Crespoa (Parmeliaceae, Ascomycota) en el nordeste de Argentina; Canoparmelia caroliniana y $C$. cryptochlorophaeanuevas citas para Argentina. Bol. Soc. Argent. Bot. 49: 161-172.

MICHLIG, A. \& L. I. FERRARO. 2012a. Nuevos registros de especies sorediadas de Parmotremacon ácido protocetrárico (Ascomycota, Parmeliaceae) en el sur de Sudamérica. Darwiniana 50: 323-331.

MICHLIG, A. \& L. I. FERRARO. 2012b. Diversidad de macrolíquenes del Parque Nacional Mburucuyá (Corrientes, Argentina). Bol. Soc. Argent. Bot. 47: 287-302.
RODRIGUEZ, J. M., HERNANDEZ, J. M., FILIPPINI, E., CAÑAS M. \& C. ESTRABOU. 2016. Nuevas citas de macrolíquenes para Argentina y ampliaciones de distribución en el centro del país. Bol. Soc. Argent. Bot. 51: 405-417.

SCUTARI, N. C. 1992. Estudios sobre Pyxinaceae foliosas (Lecanorales, Ascomycotina) de la Argentina IV: Clave de los géneros y las especies de la provincia de Buenos Aires. Bol. Soc. Argent. Bot. 28: 169-173.

SIPMAN, H. 2005. Lichen determination keys -neotropical Hypotrachyna-. Disponible en: http:// www.bgbm.org/sipman/keys/Neohypot.htm [Acceso: 20 March 2016].

Recibido el 17 de mayo de 2016, aceptado el 7 de noviembre de 2016 . 